\title{
La mejora de la competencia comunicativa en el alumnado disléxico. Un estudio de casos
}

\author{
Lorena Collados Torres. Universidad de Murcia \\ Cristina Baello Ponte. Universidad de Murcia \\ M. a Ángeles Hernández Prados. Universidad de Murcia
}

Recepción: 26.10.2019 | Aceptado: 07.11.2019

Correspondencia a través de ORCID: M. ángeles Hernández

0000-0002-3617-215X

Citar: Collados, L., Hernandez, MA. y Baello, C. (2019). La mejora de la competencia comunicativa en el alumnado disléxico. Un estudio de casos. ReiDoCrea, 8, 389-398.

Resumen: Las dificultades en la capacidad lectora son un rasgo significativo de la dislexia, provocando alteraciones en los procesos cognitivos y psicosociales de los diagnosticados, que alteran el rendimiento escolar y las relaciones interpersonales, por tanto, demanda actuaciones responsables y cualificadas por parte de los docentes. La finalidad de este trabajo es describir el proceso de evaluación, intervención y seguimiento de una secuencia de actividades para la mejora de la competencia lectora en un estudiante diagnosticado de dislexia. Concretamente, en este estudio de caso se presentan los resultados obtenidos en el diagnóstico lector de un alumno de 13 años realizado mediante la prueba PROLEC-SE, así como el programa de actividades diseñado para la mejora de la competencia lectora. Los resultados mostraron que el alumno presenta dificultades en el proceso sintáctico y en el proceso semántico, los cuales afectan directamente en la lectura. Como principal conclusión se debe destacar una mayor implicación en el proceso de enseñanza-aprendizaje y un conocimiento más ajustado de las dificultades que presenta el alumno disléxico por parte del docente.

Palabras clave: Dislexia y proceso lector

\section{The improvement of communicative competence in dyslexic students. A case study}

\begin{abstract}
Difficulties in reading ability are a significant feature of dyslexia, causing alterations in the cognitive and psychosocial processes of diagnosed student, alter school performance and interpersonal relationships, therefore, demands responsible and qualified actions by teachers. This work is intended to describe the process of evaluation, intervention and monitoring of a sequence of activities for the improvement of reading competence in a student diagnosed with dyslexia. Specifically, this case study presents the results obtained in the reader diagnosis of a 13-year-old student carried out through the PROLEC-SE test, as well as the program of activities designed to improve reading proficiency. The results showed that the student presents difficulties in the syntactic process and in the semantic process, which directly affect the reading. As a main conclusion, a greater involvement in the teaching-learning process and a more adjusted knowledge of the difficulties presented by the dyslexic student by the teacher should be highlighted.
\end{abstract}

\section{Keywords: Dyslexia and reading process}

\section{Introducción}

Leer es un aprendizaje instrumental esencial en la escolaridad de todo alumno, viéndose considerablemente afectado el rendimiento académico cuando se detectan y diagnostican dificultades en cualquiera de los procesos implicados en la lectura. La motivación y la disposición de los estudiantes de Educación Secundaria Obligatoria (ESO) es muy importante para el desarrollo del hábito lector. Son numerosas las investigaciones que indagan en la necesidad que el alumnado de esta etapa educativa tiene de orientaciones dirigidas hacia fomento de la actividad lectora a través de propuestas atractivas para su edad e inquietudes (Delgado, 2007). Sin embargo, en ocasiones, estas condiciones no son suficientes porque intervienen alteraciones en la lectura que provocan la frustración del alumnado, mostrando rechazo hacia aquello que no entienden o sobre lo que piensan no tener las suficientes habilidades (Zuppardo, Serrano y Pirrone, 2017). Por tanto, resulta significativa la intervención 
psicopedagógica con aquellos estudiantes que presentan alteraciones en los mecanismos involucrados en la práctica lectora, dado que debemos rentabilizar esfuerzos para evitar precisamente la apatía motivada por la dificultad de alcanzar las metas planteadas.

Diferentes estudios señalan la necesidad imperante de abordar esta cuestión desde la importancia que tiene la competencia lectora en los resultados obtenidos por los alumnos en distintas materias. No podemos sintetizar la cuestión en la concepción de buen lector y mal lector. No se trata de clasificar a los estudiantes en grupos determinados, sino de proporcionar estrategias que atiendan al aprendizaje a partir de variables como "el control emocional, la selección de información relevante, el pensamiento crítico y creativo, la recuperación de la información aprendida y en la planificación y la evaluación de la información como parte del proceso de metacognición" (Pinto, Martínez y Jiménez-Taracido, 2016, p. 447). Es fundamental la atención en las necesidades reales de los alumnos, en tanto que una mala gestión de estas puede propiciar sentimientos de rechazo ante la lectura, que requiere de un desenvolvimiento óptimo en la comprensión, la reflexión o la interpretación, que afectan a las diferentes áreas que conforman el currículo de la ESO.

Ante la diversidad existente en el aula y la necesidad de atender a diferentes ritmos en la configuración de lectores eficaces, resulta vital el papel del docente en las orientaciones que el alumno precisa para alcanzar los objetivos propuestos, dado que la continua exposición del educando al fracaso en la consecución de las tareas termina ocasionando desinterés a la hora de realizar las actividades indicadas (GonzálezValenzuela y Martín-Ruíz, 2016). Por ello, la formación del profesorado no puede ir dirigida únicamente a las materias que se centran en los procesos de lectura como es el caso de Lengua y Literatura. Es incuestionable la implicación de todas las especialidades que conforman la etapa educativa de ESO, pues la realidad vigente en el sistema educativo precisa de educadores que proporcionen al alumnado respuestas a las dificultades de aprendizaje enfocadas a la lectura, las cuales afectan a todas las áreas de conocimiento en las que los educandos deben de comprender directrices escritas, formuladas a través de enunciados. Estas requieren, además, la puesta en macha de habilidades que permitan la realización correcta de las tareas especificadas, por tanto, no solo se debe abordar la intervención desde la descodificación del texto, sino desde los distintos conocimientos morfológicos, sintácticos y semánticos, así como aquellos que interfieren directamente en el bagaje del alumno y en los contenidos estudiados en las diferentes disciplinas.

\section{Alumnado disléxico y dificultades lectoras}

Las dificultades del aprendizaje hacen referencia a un grupo de trastornos neurobiológicos que manifiesta alteraciones en la de adquisición de alguna capacidad como hablar, leer, escribir, entender o razonar (APA, 2013). De todas las patológicas calificadas con los problemas y dificultades lectoras, nos centramos en este trabajo en la dislexia, definida por la Clasificación Internacional de Enfermedades (CIE-10) como un déficit significativo del desarrollo de la capacidad de leer (comprensión, reconocimiento de palabras, la capacidad de leer en voz alta y el rendimiento en actividades que requieren leer).

Las principales manifestaciones sintomáticas en los adolescentes con dislexia están relacionadas con la presencia de dificultades en la precisión y fluidez en el reconocimiento de palabras escritas, con alteraciones en las habilidades de decodificación lectora y deletreo, así como con escasez de habilidades que afectan en 
la comprensión lectora (Romero, Castaño y Córdoba, 2017). Estas afectaciones no son motivadas por ninguna deficiencia en la capacidad intelectual, pero sí van intrínsecamente ligados a la expresión escrita, dado que es frecuente hallar en estos sujetos vacilaciones y errores en cuestiones gramaticales y ortográficas (Suárez, Villanueva, González y Gónzález, 2016)

Autores como Herrera y Defior (2005) señalan la hipótesis del procesamiento fonológico, como principal causa de la dislexia, el cual afecta directamente en la velocidad de denominación, el reconocimiento de las palabras y la memoria a corto plazo. Sin embargo, otros estudios (Carrillo y Alegría, 2009) consideran que además de las dificultades en el mecanismo gráfico-fonológico, es necesario considerar aquellas propias del diagnóstico que proceden de alteraciones en los mecanismos de acceso directo, que provocan deficiencias léxico-ortográficas. Así pues, en lo que respecta a los diferentes subtipos de dislexia, hay otras investigaciones que apuntan en una y otra dirección, destacando la denominada doble ruta, en la que interfieren los dos modelos anteriores (Fumagalli, Barreyro, Jacubovich, Olmedo \& Jaichenco, 2016).

La dislexia es además un trastorno que frecuentemente aparece asociado con otros déficits (Mayes, Breaux, Calhoun \& Frye, 2019), entre los que se encuentra el Trastorno por Déficit de Atención/Hiperactividad (TDAH), cuyo porcentaje de prevalencia se halla entre el $20 \%$ y el $40 \%$ de los sujetos diagnosticados (Moura et al., 2017). Distintos autores han investigado también sobre la comorbilidad que presenta la dislexia con el Trastorno Espectro Autista (2018). La convivencia de ambos trastornos en el diagnóstico de un sujeto oscila entre un 6-30\%, por lo que la asociación varía considerablemente según los resultados del estudio realizado. Otras afectaciones que pueden aparecer ligadas a la dislexia son los trastornos de conducta, trastornos de ansiedad y depresión (Hendren, Haft, Black, White \& Hoeft, 2018).

Actualmente, la dislexia es una dificultad de aprendizaje sobre la que se han llevado a cabo numerosas investigaciones, que han posibilitado la consolidación de un gran compendio de recursos que permiten el desarrollo y refuerzo académicos de aquellos estudiantes que están diagnosticados de este trastorno. Sin embargo, es necesario seguir ampliado los trabajos que abordan la dislexia desde el ámbito educativo, especialmente centrados en la formación y preparación del profesorado para que pueda actuar y atender a estos estudiantes de forma adecuada (Carrillo, 2012), dado que aunque su prevalencia ha sido establecida en un $10 \%$ de los estudiantes y convivimos con este déficit de forma generalizada, sigue resultando desconocido para numerosos docentes de la comunidad educativa (Quintanal, 2011) y es necesario aportar medidas educativas desde el rigor científico que sirvan apara mitigar las dificultades que experimentan los alumnos diagnosticados de este trastorno, que afecta direcmante en el proceso de lectoescritura.

\section{Objetivo}

El objetivo que nos planteamos con este estudio es la mejora de la competencia lectora de un alumno disléxico matriculado en segundo de Educación Secundaria Obligatoria, a través de actividades que sirva de orientación a los docentes para el desarrollo de la comprensión lectora.

1. Identificar los procesos que se ven implicados en las dificultades de comprensión lectora que presenta el alumno disléxico. 
2. Diseñar una secuencia de actividades para promover la mejora en la competencia lectora en las necesidades identificadas.

\section{Método}

Este trabajo emplea la metodología de investigación de estudio de caso, que a pesar de haber sido muy cuestionado y utilizado bajos parámetros de incertidumbre en sus inicios, actualmente se ha consolidado como una herramienta valiosa en la investigación (Martínez Carazo, 2011). Aunque el estudio de caso se sitúa en la investigación cualitativa, se ha empleado para la identificación de las dificultades del proceso lector una prueba que permite cuantificar las respuestas atendiendo al número de aciertos y errores cometidos en las pruebas, pues siguiendo a Quintanal (2011), "Toda intervención, incluso preventiva, debe responder a un diagnóstico preciso y estar debidamente planificada". Por tanto, este estudio comprende dos etapas claramente diferenciadas, una primera fase de diagnóstico y finalmente, el diseño de una secuencia de actividades como propuesta para la mejora de la competencia lectora dentro del aula.

\section{Participante - Descripción del caso}

El sujeto con el que se ha realizado el estudio cursa $2^{\circ}$ de ESO en un centro de la ciudad de Yecla, en la Región de Murcia. El alumno fue diagnosticado de dislexia en Educación Primaria, por tanto, desde este momento ha recibido diversas adaptaciones no significativas por parte del docente de Pedagogía Terapéutica (PT). No obstante, en la actualidad manifiesta cierto rechazo hacia las ayudas que se le proporcionan, pues considera que no quiere ser distinto a los demás y, por tanto, prefiere que no se realicen medidas de atención a la diversidad en su evolución académica. En lo que respecta a su familia, los padres del estudiante no presentan dislexia, por tanto, se descarta el componente genético como causa de la dislexia de la que está diagnosticado.

Es un alumno muy participativo y pone mucho interés en alcanzar los objetivos propuestos en cada una de las asignaturas. El rendimiento es bueno en la mayoría de las disciplinas, aunque en Lengua suele obtener calificaciones más bajas debido a los estándares de aprendizaje de esta materia, en los que muestra más dificultad. La relación con sus compañeros es positiva y está muy integrado en aula de referencia.

\section{Instrumentos y materiales}

Para medir las habilidades en la comprensión lectora, se ha utilizado como instrumento la batería de pruebas PROLEC-SE (Ramos y Cuetos, 1997). En concreto, se han seleccionado dos tests, uno para el proceso sintáctico y otro para el proceso semántico. El primero se corresponde con la prueba 3 , en la que aparecen 24 dibujos, acompañados de tres oraciones para que el alumno seleccione la opción correcta en relación con la representación del dibujo.

En lo que respecta a la compresión textual, se ha seleccionado el texto expositivo "Los esquimales", compuesto de 339 palabras, que pertenece a la prueba 5 del cuaderno del alumno. El sujeto tiene que leer el texto y, a continuación, debe contestar diez preguntas, las cuales pueden ser literales o inferenciales. Para la evaluación del uso de los signos de puntuación y la ortografía se ha utilizado también esta prueba. A partir de la respuesta del estudiante, se valorará la expresión escrita en la que se puede 
medir cualitativamente las habilidades y las dificultades semánticas, así como cuestiones formales de la escritura.

\section{Procedimiento}

Las pruebas administradas al alumno de forma individual se realizaron en el contexto escolar, concretamente en un aula organizada para evitar posibles distracciones que pudieran interferir en la atención y la concentración requerida para abordar las tareas. Primero se evalúo la prueba que medía el proceso sintáctico y a continuación, el que valora el proceso semántico. En ambos casos se suprime la variable del tiempo, dado que en el análisis de los resultados de la aplicación de la prueba es más relevante detectar las dificultades que presenta el alumno, más que la exactitud temporal destinada a la finalización de la tarea.

Así pues en la elaboración de la segunda actividad vinculada al componente semántico, se le permitió la utilización del material proporcionado en el cuadernillo, es decir, le facilitamos el texto que tenía que leer durante la contestación de las preguntas explícitas e inferenciales, dado que en esta investigación resultaba relevante conocer el procedimiento que el alumno empleaba en los ejercicios propuesto, con la intención de analizar otras variables medidas de forma cualitativa como la atención o la memoria.

\section{Resultados}

La evaluación se ha centrado en el rendimiento observado en las dos pruebas administrados, atendiendo a la implicación de los procesos sintácticos (Tabla 1) y semánticos (Tabla 2) en la lectura. La valoración que se ha realizado de los resultados atiende a la descripción cualitativa de los mismos, en tanto que la presente investigación se centra en un caso único, y por tanto, como señala la batería PROLEC-SE (1997, 29), es más relevante considerar los errores y aciertos en cada una de las pruebas para "la detección de problemas de lectura y no una distribución de buenos y malos lectores".

\begin{tabular}{|c|c|c|c|c|c|c|}
\hline \multicolumn{7}{|c|}{$\begin{array}{l}\text { Tabla 1. Respuesta del alumno en el emparejamien } \\
\text { oración imágen (PROLECSE, 1997) }\end{array}$} \\
\hline \multirow[b]{2}{*}{1} & \multirow[b]{2}{*}{$P$} & \multirow[b]{2}{*}{0} & \multirow[b]{2}{*}{1} & \multicolumn{3}{|l|}{$\mathbf{R}$} \\
\hline & & & & A & B & C \\
\hline 2 & OF & 0 & 1 & A & B & C \\
\hline 3 & SE & 0 & 1 & A & B & C \\
\hline 4 & RO & 0 & 1 & A & B & C \\
\hline 5 & $\mathrm{OE}$ & 0 & 1 & A & B & C \\
\hline 6 & RS & 0 & 1 & $A$ & B & C \\
\hline 7 & $P$ & 0 & 1 & A & B & C \\
\hline 8 & SE & 0 & 1 & A & B & C \\
\hline 9 & RS & 0 & 1 & A & B & C \\
\hline 10 & OF & 0 & 1 & A & B & C \\
\hline 11 & $\mathrm{OE}$ & 0 & 1 & A & B & C \\
\hline 12 & RO & 0 & 1 & A & B & C \\
\hline
\end{tabular}




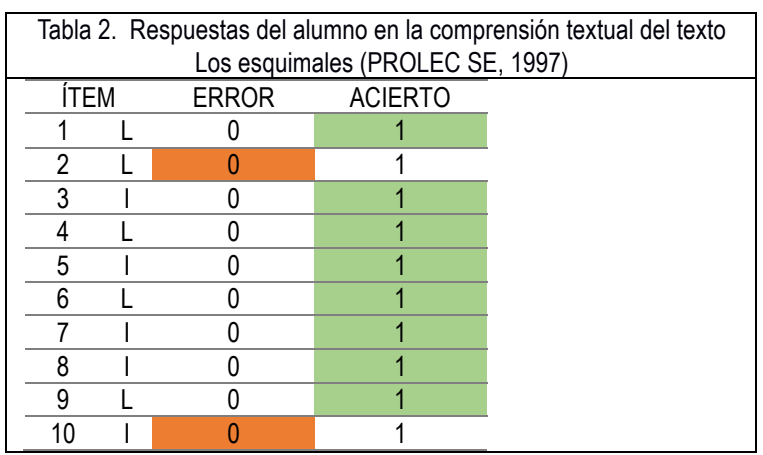

En los resultados obtenidos en la prueba de sintaxis (Tabla 3), se observan dificultades en el emparejamiento dibujo-oración, así como en el uso adecuado de los signos de puntuación. En las estructuras gramaticales de objeto escindido, como "Es al bombero a quien riega el cura", "Es al enfermo a quien mira la temperatura el médico" y "Es el mecánico a quien ayuda el policía", en la que diferenciamos dos partes a través del verbo ser y el verbo regar/mirar/ayudar, el alumno no identifica el sujeto de la acción. Hallamos errores frecuentemente en aquellos ítems que muestran esta estructura, en la que el sujeto ocupa la posición final en la oración. También encontramos respuestas incorrectas cuando el estudiante tiene que identificar las oraciones de relativo de objeto, en enunciados como "El general al que saluda el soldado está delgado", en las que no identifica el sujeto y el complemento de la oración principal.

\begin{tabular}{|cc|}
\hline $\begin{array}{c}\text { Tabla 3. Puntuación alcanzada por el alumno } \\
\text { disléxico en la prueba de sintaxis (PROLEC-SE, } \\
\text { 1997) }\end{array}$ \\
\hline \multicolumn{2}{c}{ PUNTUACIÓN TOTAL } \\
\hline \multicolumn{1}{c}{ PUNTUACIÓN MÁXIMA= 24 } \\
\hline P= \\
(PASIVAS) \\
\hline OF= \\
OBJETO \\
FOCALIZADO \\
\hline SE $=$ \\
(SUJETO \\
ESCINDIDO) \\
\hline OE $=$ \\
(OBJETO \\
ESCINDIDO) \\
\hline RO $=$ \\
(RELATIVO \\
OBJETO) \\
\hline RS $=$ \\
(RELATIVO \\
SUJETO) \\
\hline TOTAL= \\
\hline \\
\end{tabular}

En cuanto a los procesos semánticos (Tabla 4), el alumno no comete errores significativos en la comprensión y la respuesta a preguntas literales e inferenciales. No obstante, encontramos dificultades en ortografía, así como en la colocación correcta de los signos de puntuación. Además posee alteraciones en la expresión motivadas por omisiones, confusión o sustitución de palabras y letras. En la aplicación de la prueba, se observaron deficiencias en la lectura eficaz y en la comprensión textual, 
dado que el alumno de forma recurrente tenía que volver al texto para recordar la respuesta a las cuestiones formuladas.

\begin{tabular}{|l|}
\hline $\begin{array}{l}\text { Tabla 4. Puntuación alcanzada por el alumno disléxico } \\
\text { en la prueba de semántica (PROLEC-SE, 1997) }\end{array}$ \\
\hline \multicolumn{2}{c|}{ PUNTUACIÓN TOTAL } \\
\hline \multicolumn{2}{c|}{ PUNTUACIÓN MÁXIMA= 10 } \\
\hline $\begin{array}{l}\text { PREGUNTAS } \\
\text { LITERALES }\end{array}$ \\
$\begin{array}{c}\text { PREGUNTAS } \\
\text { INFERENCIALES }\end{array}$ \\
\hline TOTAL=
\end{tabular}

\section{Secuencia de actividades para la intervención educativa}

La intervención está compuesta de una secuencia de seis actividades, para cuya elaboración se han tenido en cuenta los errores que el alumno ha tenido en las dos pruebas suministradas. A continuación, se establece una relación entre los objetivos, la descripción de las actividades que se han planteado, así como el tiempo destinado a la realización de cada una de ellas. 


\begin{tabular}{|c|c|c|c|}
\hline Secuencia didáctica & Temporalización & Objetivos & Descripción de actividades \\
\hline $\begin{array}{c}\text { Actividad } 1 \\
\text { Añadiendo elementos }\end{array}$ & 5 & $\begin{array}{ll}\text { - } & \text { Comprender el significado de cada oración debido al cambio estructural. } \\
\text { - } & \text { Comprender por qué influye el contexto en el significado. } \\
\text { - } & \text { Identificar el verbo, sujeto y predicado de oración junto a sus complementos verbales y su } \\
\text { estructura en las oraciones: complemento directo y complemento circunstancial. }\end{array}$ & $\begin{array}{l}\text { Construcción, a partir de una oración simple, } \\
\text { de diferentes estructuras a través de la inclusión de } \\
\text { distintas categorías gramaticales y funciones sintácticas. }\end{array}$ \\
\hline La historia dada e inventada & $15^{\prime}$ & $\begin{array}{l}\text { Producir un escrito para fomentar la capacidad de escritura. } \\
\text { Fortalecer la fluidez y el gusto por la lectura. } \\
\text { Emplear signos de puntuación. } \\
\text { Respetar las reglas ortográficas. }\end{array}$ & $\begin{array}{l}\text { Elaboración de un cuentro tradicional } \\
\text { partiendo de un texto dado, en el que hay que tener en } \\
\text { cuenta las características propias del género y mantener } \\
\text { la coherencia en las propiedades de los personajes, el } \\
\text { espacio y el tiempo proporcionado previamente. }\end{array}$ \\
\hline $\begin{array}{l}\text { Actividad } 3 \\
\text { Entiendo lo que leo }\end{array}$ & $15^{\prime}$ & $\begin{array}{ll}\text { - } & \text { Evaluar la comprensión a través de preguntas inferenciales. } \\
\text { - } & \text { Evaluar la capacidad del alumno para completar las preguntas. realizadas con ideas } \\
\text { relevantes del texto que han tenido que leer previamente. }\end{array}$ & $\begin{array}{l}\text { Lectura comprensiva del texto y respuesta } \\
\text { de las cuestiones formuladas en relación con el } \\
\text { contenido de la historia. }\end{array}$ \\
\hline Cualidades analizadas & 5 & $\begin{array}{ll}\text { - } & \text { Explicar de manera adecuada, detallada y ordenada cómo es el personaje. } \\
\text { - } & \text { Entender por qué la relación entre palabras afecta a la comprensión. } \\
\text { - } & \text { Identificar el verbo, sujeto y predicado de oración junto a sus complementos verbales y su } \\
\text { estructura en las oraciones: atributo y complemento directo. }\end{array}$ & $\begin{array}{l}\text { - Diseño de la descripción de un personaje y } \\
\text { análisis sintáctico de los enunciados que constituyen el } \\
\text { texto. }\end{array}$ \\
\hline $\begin{array}{c}\text { Actividad } 5 \\
\text { Significados escogidos }\end{array}$ & 5 & $\begin{array}{ll}\text { - } & \text { Comprender el significado y sentido de signos lingüísticos (palabras). } \\
\text { - } & \text { Entender por qué la relación entre palabras afecta a la comprensión. }\end{array}$ & $\begin{array}{l}\text { - Construcción de campos semánticos a partir } \\
\text { de palabras dadas, utilizando el vocabulario del texto y } \\
\text { añadiendo otras aportaciones propias. }\end{array}$ \\
\hline Crear con palabras e imágenes & $10^{\prime}$ & $\begin{array}{ll}\text { - } & \text { Fortalecer la comprensión lectora a través de la estrategia de creación de un cómic. } \\
\text { - } & \text { Expresar ideas a través de dibujos como medio para valorar el conocimiento y la } \\
\text { comprensión del alumno. } \\
\text { - } \\
\text { - }\end{array}$ & $\begin{array}{l}\text { - Creación de un cómic siguiendo la historia } \\
\text { previa y producción de los diálogos de los personajes } \\
\text { atendiendo a las cualidades y acciones que los } \\
\text { caracterizan. }\end{array}$ \\
\hline
\end{tabular}




\section{Discusión}

En este estudio, presentamos las dificultades del proceso lector del caso de un sujeto de 13 años en relación con los procesos sintácticos y semánticos que intervienen, teniendo en cuenta que el estudiante está diagnosticado de dislexia. Además, se propone la realización de una secuencia de actividades, que permite abordar las dificultades que el alumno presenta en las dos pruebas seleccionadas, con la finalidad de promover su mejora. Somos conscientes de las limitaciones y dudas que despiertan este tipo de estudios en la investigación educativa, la mayoría sustentadas en la dificultad de generalización de los resultados obtenidos, siendo más comunes, curiosamente, los estudios de casos en el ámbito clínico. No obstante, la credibilidad de las conclusiones obtenidas se basa, en última instancia, en la calidad misma de la investigación desarrollada (Martínez Carazo, 2011, p.173) y no tanto en las posibilidades de generalización.

Algunos estudios previos de casos se centran exclusivamente en la fase de diagnostico describiendo las dificultades asociadas a la dislexia y las características personales y familiares (Salgado, et al, 2006; Rasposo-Rivas y Salgado, 2017). En esta misma línea, se han expuesto los resultados obtenidos de la aplicación del PROLEC-SE (1997), en los que se ha tenido en cuenta cualitativamente los errores que el estudiante ha mostrado en la medición del componente sintáctico y semántico, El alumno presenta dificultades en el análisis de las oraciones de objeto escindido, en el que no precisa correctamente quién es el sujeto que realiza la acción en la asociación oración-imagen. Así mismo, en la significación del contenido del texto y en la respuesta a las preguntas explícitas e inferenciales, el estudiante muestra alteraciones en la escritura en lo que respecta al uso de los signos de puntuación, en el orden de letras o la omisión de estas, en una palabra, además de problemas para focalizar la atención y recordar una vez leído el texto el contenido para cumplimentar el ejercicio.

Sin embargo, la principal fortaleza del trabajo expuesto no radica tanto en los resultados obtenidos, sino más bien en la secuencia de actividades diseñada. Somos conocedoras del interés y demanda de este tipo de estudios por parte de la comunidad educativa (Ripoll y Aguado, 2016; Sánchez, Martín, Moreno y Espada, 2018; Ferrada y Outón, 2019).

Los hallazgos descritos aquí nos revelaron la importancia de trabajar educativamente estos contenidos con los estudiantes disléxicos, a pesar de que estudios recientes presenten resultados poco esperanzadores. Al respecto, Maehler, Joerns y Schuchardt (2019) desvelaron una visión pesimista de la mejora de rendimiento de la memoria de trabajo en alumnos con y sin dislexia a través de un programa de capacitación.

Esperamos que el caso descrito ayude a entender a los docentes la importancia de recibir formación especifica sobre las dificultades lectoras, de trabajar interdisciplinar y comunitariamente con otros agentes y contextos, y de implicarse en la implementación de medidas y estrategias que ayuden a solventarlas o mitigarlas. A pesar de que son muchos los estudios que, tras un análisis exploratorio de necesidades, esbozan propuestas de intervención, consideramos una limitación de este estudio la no aplicación de la secuencia de actividades, que permitirá valorar la eficacia e impacto en el alumnado con dislexia. 


\section{Referencias}

Asociación Americana de Psiquiatría. (2013). Manual diagnóstico y estadístico de los trastornos mentales (DSM-5). Madrid: Editorial Médica Panamericana.

Carrillo, M. y Alegría, J. (2009). Mecanismos de identificación de palabras en niños disléxicos en español: ¿Existen subtipos?. Ciencias Psicológicas, 3(2), 135-152.

Delgado, B. (2007). Fundamentos del proceso lector. Motivar la lectura en la Educación Secundaria. Ocnos: Revista de Estudios sobre Lectura, (3), 39-53.

Ferrada, N. y Outón, P. (2019). Eficacia de un programa de fluidez lectora con escolares disléxicos. Revista de Logopedia, Foniatría y Audiología, 39(1), 11-19.

Fumagalli, J., Barreyro, J. P., Jacubovich, S., Olmedo, A., \& Jaichenco, V. (2016). Phonological skills, reading accuracy and speed in Dyslexic patients/Habilidades fonoaudiológicas, precisão leitora e velocidade em pacientes com dislexia. Panamerican Journal of Neuropsychology, 10(1), 72-87.

González-Valenzuela, M. J. y Martín-Ruíz, I. (2016). Motivation and written language in Spanish adolescents. Anales De Psicología / Annals of Psychology, 32(2), 466-473.

Hendren, R. L., Haft, S. L., Black, J. M., White, N. C., \& Hoeft, F. (2018). Recognizing psychiatric comorbidity with reading disorders. Frontiers in Psychiatry, 9 (101), 1-10.

Herrera, L. y Defior, S. (2005). Una aproximación al procesamiento fonológico de los niños prelectores: conciencia fonológica, memoria verbal a corto plazo y denominación. Psykhe, 14(2), 81-95.

Maehler, C., Joerns, C., \& Schuchardt, K. (2019). Training Working Memory of Children with and without Dyslexia. Children, 6 (3), 47

Mayes, S. D., Breaux, R. P., Calhoun, S. L., \& Frye, S. S. (2019). High Prevalence of Dysgraphia in Elementary Through High School Students With ADHD and Autism. Journal of Attention Disorders, 23(8), 787-796.

Martínez Carazo, P. C. (2011). El método de estudio de caso Estrategia metodológica de la investigación científica. Revista científica Pensamiento y Gestión, (20).

Mejía, C. y Varela, V. (2015). Comorbilidad de los trastornos de lectura y escritura en niños diagnosticados con TDAH. Psicología desde el Caribe, 32(1), 121-143.

Moura, O., Pereira, M., Alfaiate, C., Fernandes, E., Fernandes, B., Nogueira, S., ... \& Simões, M. R. (2017). Neurocognitive functioning in children with developmental dyslexia and attention-deficit/hyperactivity disorder: Multiple deficits and diagnostic accuracy. Journal of Clinical and Experimental Neuropsychology, 39(3), 296-312.

Quintanal Diías, J. (2011). La dislexia: desconocida, cuando no, ignorada. Padres y maestros, (340), 16-19.

Pinto, N., Martínez, A. I. y Jiménez-Taracido, L. (2016). Estrategias de aprendizaje, comprensión lectora y rendimiento académico en Educación Secundaria. Psicologia Escolar e Educacional, 20(3), 447-456.
Ramos, J. L. y Cuetos, F. (1997). PROLEC-SE. Evaluación de los procesos lectores. Madrid: TEA

Ramos, J.L. y Galve, J.L. (2017). Dificultades específicas de lectoescritura. Vol. 1. Modelo teórico, evaluación e intervención en la lectura y las dislexias. Madrid. ZP.

Ripoll, J. C. y Aguado, G. (2016). Eficacia de las intervenciones para el tratamiento de la dislexia: una revisión. Revista de Logopedia, Foniatría y Audiología, 36(2), 85-100.

Romero, A., Castaño, C. y Córdoba, M. (2017). Eficacia de un programa de intervención temprana para reducir las señales de riesgo de la dislexia. Revista de Educación Inclusiva, 9(2), $186-200$

Salgado, C. A. et al. (2006). Avaliação fonouaudiológica e neuropsicológica na dislexia do desenvolvimento do tipo mista: relato de caso. Salusvita, 25 (1), 91-103.

Sánchez, S., Martín, R. A., Moreno, I. y Espada, R. M. (2018). Revisión sobre la intervención precoz en dificultades de aprendizaje relacionadas con la lectura. Revista Electrónica Interuniversitaria de Formación del Profesorado, 21(3), 35-45.

Suárez, P., Villanueva, N., González, S. y Gónzález, M. (2016). Dificultades de escritura en niños españoles con dislexia. Infancia y Aprendizaje: Journal for the Study of Education and Development, 39(2), 291-311.

Zuppardo, L., Serrano, F. y Pirrone, C. (2017). Delimitando el perfil emotivo-conductual en niños $y$ adolescentes con dislexia. Revista RETOS XXI, 1(1), 88-104. 